OPEN ACCESS

Edited by:

Domenico Tricarico,

University of Bari Aldo Moro, Italy

Reviewed by:

Lishuang Cao,

Vertex Pharmaceuticals

(United Kingdom), United Kingdom

Isaac Jardin,

University of Extremadura, Spain

*Correspondence:

Millet Treinin

millet.treinin@mail.huji.ac.il

Specialty section:

This article was submitted to

Pharmacology of Ion Channels

and Channelopathies,

a section of the journal

Frontiers in Pharmacology

Received: 19 August 2019

Accepted: 03 December 2019

Published: 17 January 2020

Citation:

Cohen E, Kumar R, Zinger T, Priel A and Treinin M (2020) GTL-1, a Calcium Activated TRPM Channel,

Enhances Nociception.

Front. Pharmacol. 10:1567. doi: 10.3389/fphar.2019.01567

\section{GTL-1, a Calcium Activated TRPM Channel, Enhances Nociception}

\author{
Emiliano Cohen ${ }^{1}$, Rakesh Kumar ${ }^{2}$, Tal Zinger ${ }^{1}$, Avi Priel $^{2}$ and Millet Treinin ${ }^{1 *}$ \\ ${ }^{1}$ Department of Medical Neurobiology, Hadassah-Medical School, Jerusalem, Israel, 2 Faculty of Medicine, School of \\ Pharmacy, The Institute for Drug Research, Hebrew University, Jerusalem, Israel
}

C. elegans PVD neurons are conserved for morphology, function and molecular determinants with mammalian polymodal nociceptors. Functions of polymodal nociceptors require activities of multiple ion channels and receptors including members of the TRP family. GTL-1, a member of the TRPM subclass of TRP channels, was previously shown to amplify PVD-mediated responses to optogenetic stimuli. Here we characterize effects of GTL-1 on PVD-mediated behavioral responses to noxious stimuli. We show that GTL-1 is required within PVD for the immediate and enduring response to thermal (cold) stimuli. But, find no significant reduction in percent animals responding to single or to repeated noxious mechanical stimuli. Nevertheless, PVD specific knockdown of $\mathrm{gtl}$-1 expression reduces the magnitude of responses to noxious mechanical stimuli. To understand GTL-1's mechanism of action we expressed it in HEK293 cells. Our results show GTL-1-dependent currents induced by activation of a Goq-coupled Designer Receptor Exclusively Activated by Designer Drugs (DREADD). In addition, using excised patches we show that GTL-1 can be activated by internal calcium. Our results are consistent with indirect, calcium dependent, activation of GTL-1 by noxious stimuli. This mechanism explains the GTL-1-dependent amplification of responses to multiple stimuli optogenetic and sensory in PVD.

Keywords: TRPM, C. elegans, nociceptors, calcium, behavior

\section{INTRODUCTION}

Responding appropriately to noxious, potentially injurious or injurious signals is essential for survival. This response requires polymodal nociceptors, multi dendritic sensory neurons innervating the skin or internal organs and responding to high threshold thermal and mechanical stimuli and to noxious chemicals (Woolf and Ma, 2007; Smith and Lewin, 2009). C. elegans PVD neurons are multi dendritic neurons, conserved for structure, function, and molecular determinants with mammalian polymodal nociceptors (Chatzigeorgiou et al., 2010; Albeg et al., 2011). These neurons respond to high threshold mechanical stimuli and to cold temperatures, responses mediated, respectively, by the DEG/ENaC channel subunits, MEC-10 and DEGT-1, and the TRPA channel, TRPA-1 (Chatzigeorgiou et al., 2010).

Transcriptomic analysis of PVD neurons identified multiple ion channel subunits likely to express in these neurons (Smith et al., 2010). Several of these channel subunits were examined for their effects on the behavioral response to optogenetic activation of PVD (Husson et al., 2012); this study identified GTL-1 as an ion channel needed within PVD for amplification of the behavioral 
response to optogenetic stimuli. GTL-1, Gon-Two Like protein, belonging to the TRPM subfamily of the TRP superfamily was previously shown to function together with GON-2 in maintaining $\mathrm{Mg}^{2+}$ homeostasis in the C. elegans intestine, as well as in controlling $\mathrm{IP}_{3}$-dependent $\mathrm{Ca}^{2+}$ oscillations to control defecation (Teramoto et al., 2005; Kwan et al., 2008; Xing et al., 2008; Xing and Strange, 2010). However, roles of GTL-1 in responses to PVD-mediated, physiological, noxious stimuli have not been studied.

Members of the TRP superfamily play key roles in nociception (Clapham, 2003; Dhaka et al., 2006; Julius, 2013). TRP proteins form nonspecific cation channels shown to respond to noxious heat, noxious cold, $\mathrm{pH}$, and mechanical stimuli. For example, TRPV1, also known as the capsaicin receptor, is expressed in mammalian nociceptors and is important for mediating responses to heat, acid and vanilloid toxins (Caterina and Julius, 1999; Caterina et al., 2000); TRPM3 functions as a noxious heat sensor (Vriens et al., 2011); and TRPM8 functions as a cold sensor (Dhaka et al., 2007). Functions of TRPs in polymodal nociceptors are not limited to nociception as they were also shown to modulate nociceptor activity. For example, in the Drosophila DA-IV polymodal nociceptors, TRP channels functioning downstream to hedgehog signaling sensitize responses to noxious stimuli (Babcock et al., 2009). Indeed, several TRPs, including TRPM channels, were shown to be activated by intracellular $\mathrm{Ca}^{2+}$, suggesting that in certain cases

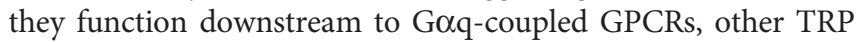
channels, or voltage activated calcium channels (Launay et al., 2002; Zurborg et al., 2007).

Here, we examine whether, GTL-1-dependent amplification of optogenetic stimuli (Husson et al., 2012) represents a physiological role for this channel in enhancing responses to noxious stimuli. For this, we examined effects of loss-of-function mutation or PVD-specific knockdown of this gene on PVDdependent responses to thermal and mechanical stimuli. Results of this analysis show defects in these responses, supporting a role for GTL-1 in PVD-mediated responses. In addition, we expressed GTL-1 in HEK293 cells to show that, like other TRPM channels, it is activated by internal calcium and can function downstream to Goq-coupled GPCRs.

\section{MATERIALS AND METHODS}

\section{Strains}

The wild type is N2. The gtl-1(ok375) mutation is a loss-offunction mutation due to a deletion of $2.7 \mathrm{~Kb}$ encoding for all the transmembrane domains of GTL-1, the strain carrying this mutation is referred to as $g t l-1(l f)$. The trpa-1(ok999) mutation encodes a loss-of-function mutation of trpa-1 due to a deletion of $1.3 \mathrm{~Kb}$, encoding for most of the intracellular N-terminal domain. The mec-4(1611) mutation is a gain-of-function mutation leading to degeneration of the six touch receptor neurons (AVM, ALM, PVM, PLM). This strain was crossed with the $g t l-1(l f)$ strain for the high threshold mechanical stimulation assay, and also served as a control in that assay. The integrated
ser-2prom3::deg-3(N293I) transgene which eliminates PVDs via degeneration (-P animals), was previously described in (Albeg et al., 2011).

\section{Plasmids}

To knockdown expression of $g t l-1$ in PVD we used the method developed in (Esposito et al., 2007) for expressing double stranded RNA of genes of interest in specific cells. Specifically, coding inserts of gtl-1 were amplified from the C. elegans RNAi library (Source BioScience, clone IV-6A13,(Kamath et al., 2003)) using primers L4440F (5’ CGACGGTATCGATAAGCTTGAT'3) and L4440R ('5- CGACGGTATCGATAAGCTTGAT-'3) each containing a HindIII site. The resulting $1.1 \mathrm{~Kb}$ fragment was cloned in both orientations downstream to a $1.7 \mathrm{~Kb}$ ser2 prom3 promoter (expressing in PVD and OLL (Tsalik et al., 2003)) in the pBluescript SKII(-) HindIII site. The plasmids expressing both orientations were injected at $50 \mathrm{ng} / \mu \mathrm{l}$ each with a ser-2prom3::GFP plasmid as a marker into wild-type $\mathrm{N} 2$ animals.

A full length cDNA of gtl-1 cloned into pGEM-T was a kind gift from Howard Baylis (University of Cambridge). For expression of GTL-1 in HEK293 cells, a 5kb KpnI-BamH1 cDNA fragment containing gtl-1 was cloned into pCDNA4/TO.

\section{Behavioral Analysis}

\section{Response to Cold Temperature Following Prolonged Exposure}

The assay was performed as previously described (Albeg et al., 2011; Cohen et al., 2014). In brief, for movement analysis animals were transferred as L4 larvae to fresh plates and grown overnight to adulthood at $20^{\circ} \mathrm{C}$. Prior to locomotion analysis single adults were transferred to a fresh NGM plate preequilibrated at $20^{\circ} \mathrm{C}$ or $15^{\circ} \mathrm{C}$ and having a thin layer of OP50 bacteria as food (overnight growth) and allowed to acclimate for $10 \mathrm{~min}$ at $20^{\circ} \mathrm{C}$ or $15^{\circ} \mathrm{C}$. Movement of each animal was then recorded at a $25 \times$ magnification and at a rate of 10 frames per second. Animals were recorded for $60 \mathrm{sec}$ or until they moved out of the frame. These movies were analyzed using software developed for this purpose [(Albeg et al., 2011), detailed description of this software is provided in http://www.cs.huji. ac.il/ feit/worms/user-manual.pdf] .

\section{Immediate Response to Cold}

For rapid and precise temperature control of a thin agar surface on which the animal's movement is recorded we developed the following experimental setup: one animal was transferred to a small piece of NGM agar. To keep the animal within the imaging field it was placed on a dot (approximately $2 \mathrm{~mm}$ in diameter) of bacteria. The agar was placed on a cover slip and put on top of a thermoelectric plate (peltier, European Thermodynamics ET127-20-25) and a thermocouple unit (National Instruments TC01) was inserted in the agar. The peltier unit was connected to a programmable power supply unit (Thurlby Thandar Instruments PL-155-P) and a digital microscope (DinoLite Pro AM413T). The power supply unit, the thermocouple unit and the microscope were connected to a PC running a Matlab program that can record the movement of the worm, receive the information from the thermocouple and adjust the voltage of 
the power supply, thus, maintaining or changing the incubation temperature.

The automated protocol to test the effects of cooling on animal behavior was as follows. First the temperature of the agar was equilibrated to $20^{\circ} \mathrm{C} .10 \mathrm{~min}$ after this temperature was achieved and stabilized, the temperature was dropped to $15^{\circ} \mathrm{C}$ or maintained at $20^{\circ} \mathrm{C}$ for control purposes. The animal remained at this temperature for $10 \mathrm{~min}$, followed by a return of the temperature to $20^{\circ} \mathrm{C}$. Recording and analysis of control and temperature drop experiments were randomized and blind to the animal's genotype. Using this imaging setup we were only able to examine reversal frequency (direction changes from forward to backward movement) and this analysis was done manually. Reversals were counted for a period of $30 \mathrm{sec}$ just before the temperature drop, immediately after the temperature reached $15^{\circ}$ $\mathrm{C}$, after $10 \mathrm{~min}$ at $15^{\circ} \mathrm{C}$, immediately after the temperature returned to $20^{\circ} \mathrm{C}$, or at the same time frames for control animals maintained at $20^{\circ} \mathrm{C}$,

\section{Mechanical Stimuli}

For response to high threshold mechanical stimulus young adult (picked as L4 to fresh plates, 10 animals per plate, and grown at $20^{\circ} \mathrm{C}$ overnight) $m e c-4(e 1611)$ and $m e c-4(e 1611)$; gtl-1(ok375) animals were examined for their response to prodding with a platinum wire pick. Animals reversing or increasing their forward speed were considered as having responded (Way and Chalfie, 1989). To test adaptation to high threshold mechanical stimulus, young adults (picked as L4 to fresh plates and grown at $20^{\circ} \mathrm{C}$ overnight) $\mathrm{N} 2, \mathrm{gtl}-1$ (ok375), and -PVD animals were examined for their response to prodding with a platinum wire pick every $10 \mathrm{sec}$ (Husson et al., 2012).

Prolonged response to noxious mechanical stimulus (transfer with a wire pick) was examined as described in (Cohen et al., 2012). Briefly, animals were grown at $20^{\circ} \mathrm{C}$ on standard nematode growth media (NGM) plates seeded with the $E$. coli strain OP50. For each assay, L4 animals were transferred for overnight growth to fresh NGM plates and examined as young adults. At the start of each experiment a single animal was transferred with a platinum wire pick to a fresh newly seeded NGM plate and worm tracking was initiated at the moment that the animal's image was captured, up to half a minute following transfer. All movies track the animals for $20 \mathrm{~min}$.

\section{Cell Culture (HEK293T) and Transfection}

Cell culture and transfection were performed as described (Geron et al., 2018). Briefly, human embryonic kidney 293T (HEK293T) cells were transfected with a total of $1 \mu \mathrm{g}$ of DNA (GTL-1 pCNA4/TO alone or GTL-1 pCNA4/TO + hM3D(Gq) (a gift from Bryan Roth (Addgene plasmid \# 45547; Gq/Dreadd)) and with pCDNA3.1(+) using Mirus LT1 transfection reagent (Mirus Bio, Madison, WI) according to manufacturer's protocol. Co-transfection with EGFP in the pCDNA3.1(+) vector was carried for quick identification of successful transfection. Transfections were performed in 12-well plates containing $3 \times 10^{5}$ cells $48 \mathrm{~h}$ before analysis. Cells were plated on $0.05 \mathrm{mg} /$ $\mathrm{ml} \mathrm{PDL}$-coated glass coverslips $(12 \mathrm{~mm})$ and incubated at $37^{\circ} \mathrm{C}$ (5\% CO2) for at least $16 \mathrm{~h}$ before electrophysiological analysis.

\section{Patch Clamp Recordings}

Voltage-clamp recordings from transfected HEK293T cell were carried out as previously described (Kumar et al., 2017; Geron et al., 2018). Briefly, membrane currents were recorded under the voltage-clamp using an Axopatch 200B patch-clamp amplifier (Molecular Devices, Sunnyvale, CA, USA). Membrane currents were digitized using a Digidata 1440A interface board and pCLAMP 10.6 software (Molecular Devices, Sunnyvale, CA, USA) with sampling frequency set to $5 \mathrm{kHz}$ and were low-pass filtered at $2 \mathrm{kHz}$. Holding voltage was $-40 \mathrm{mV}$ for transfected HEK293T. Patch electrodes were fabricated from borosilicate glass using the P1000 Micropipette Puller (Sutter Instrument) and fire-polished using the Microforge MF-900 (Narishige, Japan) to a resistance of 2-4 $\mathrm{M} \Omega$ for both the inside-out and perforated patch recordings.

The inside-out configuration was performed as previously described (Kumar et al., 2017). Briefly, both the pipette solution and intracellular solutions constituted (mM): $150 \mathrm{NaCl}, 2.8 \mathrm{KCl}, 2$ $\mathrm{CaCl}_{2}$, and 10 HEPES, adjusted to $\mathrm{pH} 7.4$ with $\mathrm{NaOH}$. Following the establishment of the excised inside-out multichannel patch, patches were continuously superfused with extracellular solutions with or without calcium via the ValveBank perfusion system (AutoMate Scientific, Berkeley, CA, USA).

The perforated-patch configuration was carried out as previously described (Priel and Silberberg, 2004; Kumar et al., 2017). Briefly, the pipette solution constituted (mM): $75 \mathrm{~K}_{2} \mathrm{SO}_{4}, 55$ $\mathrm{KCl}, 5 \mathrm{MgSO}_{4}, 10 \mathrm{HEPES}$, adjusted to $\mathrm{pH} 7.2$ with $\mathrm{KOH}$. Nystatin (Sigma, St. Louis, MO, USA) was used for patch perforation at a working concentration of $200 \mu \mathrm{M}$. To this end, it was dissolved in Dimethyl Sulfoxide (DMSO; Sigma, St. Louis, MO, USA) to obtain a $55 \mathrm{mM}$ stock solution, which following $1 \mathrm{~min}$ ultra-sonication was diluted in pipette solution to obtain working solution. Nystatin solutions were freshly prepared in the dark every $2 \mathrm{~h}$. Only cells with series resistance of $\leq 15 \mathrm{M} \Omega$ were used for analysis. The extracellular solution contained (mM): $140 \mathrm{NaCl}, 2.8 \mathrm{KCl}, 2$ $\mathrm{MgSO}_{4}, 1.8 \mathrm{CaCl}_{2}, 10 \mathrm{HEPES}, 10 \mathrm{D}$-glucose, adjusted to $\mathrm{pH} 7.4$ with $\mathrm{NaOH}$ (Ringer solution). Once the perforated-patch or the standard whole cell configuration was established, cells were continuously superfused with extracellular solutions. To avoid effects from pre-exposure of the recorded cells to the applied agonist, cells were exposed to them once, allowing the recording of a single cell from each coverslip.

\section{Statistical Analysis}

Statistical analyses were performed using the Matlab statistical analysis toolbox and Prism 7 (GraphPad Software, La Jolla, CA, USA). Student's t-test and ANOVA were used to determine statistical significance. Electrophysiological analysis was performed by using pClamp 10.6 software.

\section{RESULTS}

\section{GTL-1 Participates in the Response to Cold Temperatures}

Previous analysis showed that PVD neurons respond to temperature downshift via the TRP channel, TRPA-1, as 
demonstrated by findings showing that trpa-1(lf) animals have an impaired response to rapid cooling. Specifically, in response to cooling trpa-1(lf) animals show no calcium transients within PVD and no increase in omega turns when examined in a liquid drop (Chatzigeorgiou et al., 2010). We have previously shown that TRPA-1 is required for the altered locomotion of animals at cold $\left(15^{\circ} \mathrm{C}\right)$ relative to warm $\left(20^{\circ} \mathrm{C}\right)$ temperatures (Cohen et al., 2014). To examine the role of GTL-1 in this behavioral response we used the same locomotion assay (Cohen et al., 2014). At $20^{\circ} \mathrm{C}$, wild-type (N2) animals moved at an average speed of $0.225 \mathrm{~mm} / \mathrm{sec}$ and had a low rate of pauses and direction reversals (Figure 1). After 10 min at $15^{\circ} \mathrm{C}$ wild-type (N2) animals' speed was significantly reduced and the rate of pauses was significantly increased relative to $20^{\circ} \mathrm{C}$ (Figures 1A, B, black bars vs. grey bars). In gtl-1 mutants effects of temperature on speed were smaller, although significant; no significant effect of temperature on speed was detected in trpa-1 mutants; and, no significant effect of temperature on the rate of pauses was seen in gtl-1 and trpa-1 loss of function mutants (Figures 1A, B). Temperature affected the rate of reversals similarly to its effect on the rate of pauses, but, in none of the strains was this effect significant (Figure 1C). The similar effects of GTL-1 and TRPA-1 in reducing the response to cold temperature relative to the response of wild-type animals are consistent with GTL-1, like TRPA-1, being required for this response (Figures 1A, B). We note that $g t l-1(l f)$ also affects locomotion at $20^{\circ} \mathrm{C}$; having significantly reduced speed and increased rate of pauses relative to wild-type (N2) animals ( $\mathrm{p}<$ 0.0001 two-way ANOVA). However, contribution of genotype to the variance in locomotion is only significant when examining the rate of pauses (two-way ANOVA, $\mathrm{p}<0.0001$ ).

\section{Effects of GTL-1 on the Response to Cold Temperature Require Its Expression in PVD}

Results in Figures 1A-C show altered locomotion of gtl-1(lf) animals at normal growth temperature $\left(20^{\circ} \mathrm{C}\right)$. Such differences may mask the effect of temperature reduction on locomotion. Moreover, $g t l-1$ is widely expressed and some of its effects may not depend on PVD expression. To examine whether the reduced response to cold temperatures depends on function of GTL-1 in PVD cells, we specifically knocked down its expression in PVD neurons using cell specific dsRNA-mediated knockdown (Esposito et al., 2007). Indeed, results in Figures 1D, E show that effects of GTL-1 on locomotion at $20^{\circ} \mathrm{C}$ do not depend on its
A

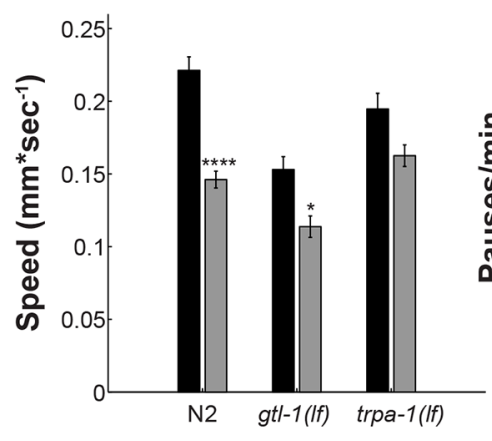

D

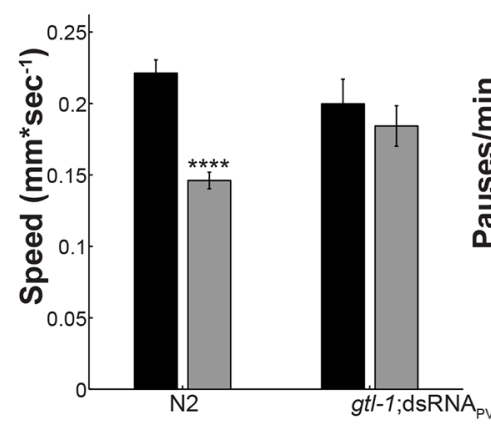

B

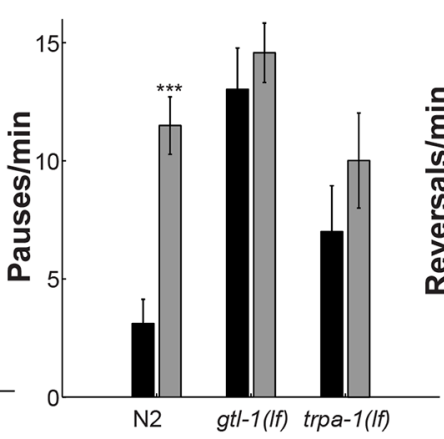

E

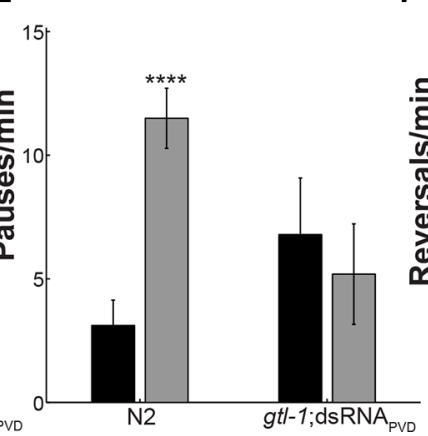

$\mathrm{F}$
C
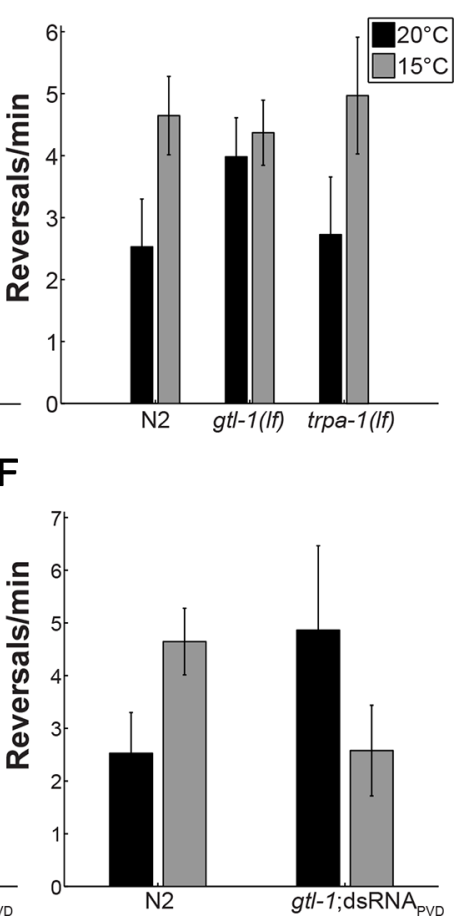

FIGURE 1 | GTL-1 is required within PVD for the effects of temperature on locomotion. Effects of temperature on locomotion were compared between wild type (N2), gtl-1(If), and trpa-1(If) animals (A-C) and between wild-type (N2) animals and animals expressing gtl-1 dsRNA specifically in PVD (gtl-1; dsRNAPVD) (D-F), at 20 ${ }^{\circ}$ C (black bars) or $15^{\circ} \mathrm{C}$ (grey bars). Number of animals, 24, 36, 29, 22, 18, and 15 (A-C) and 24, 36, 13, and 12 (D-F) in order of appearance. Significant differences for the same strain at $15^{\circ} \mathrm{C}$ relative to $20^{\circ} \mathrm{C}$ were examined using two-way ANOVA with bonferroni's multiple comparisons correction $\left({ }^{*}-p<0.05\right.$, ${ }^{* \star \star}$ - $p<0.01$,

$\left.{ }^{* \star \star \star}-p<0.0001\right)$. Temperature contributes significantly to the variance in $A(p<0.0001), B(p=0.0018), C(p=0.0112), D(p<0.0001)$ and $E(p=0.042) ; g e n o t y p e$ contributes significantly to the variance only in $B(p<0.0001)$; and effects of the interaction are significant in $A(p=0.0069), B(p=0.0476), D(p=0.0069), E(p=$ $0.0032)$, and $F(0.026)$. 
expression in PVD. Moreover, genotype does not significantly contribute to the variance in locomotion when comparing wildtype animals to animals in which $g t l-1$ was specifically silenced in PVD (two-way ANOVA). Importantly, effects of cold temperature on locomotion are eliminated in the transgenic animals in which gtl-1 was specifically knocked-down in PVD. In addition, the residual effects of temperature on locomotion speed seen in $g t l-1(l f)$ animals are not seen when $g t l-1$ is specifically silenced in PVDs (compare Figures 1A-D). Thus, GTL-1's function in PVD is required for the behavioral response of C.elegans to cold temperatures while effects of GTL-1 on locomotion under normal growth temperature are independent of PVD expression.

\section{Effects of GTL-1 on the Immediate Response to Cold}

Results described above (Figure 1) show locomotion following adaptation to cold temperatures $\left(10 \mathrm{~min}\right.$ after transfer to $\left.15^{\circ} \mathrm{C}\right)$ and may also include an enduring response to transfer, a strong mechanical stimulus (Cohen et al., 2012). To examine whether gtl-1 participates in the immediate response to temperature downshift we used a setup that allows us to rapidly cool the agar surface on which the animals is placed while recording the animal's locomotion (see Methods). Due to poor image quality in this setup movies were manually analyzed. Therefore, only one parameter was tested, the number of reversals. Reversals were counted during a $30 \mathrm{sec}$ interval immediately before temperature downshift and immediately after temperature downshift and compared to the number of reversals in animals that were continuously maintained at $20^{\circ} \mathrm{C}$. To reduce effects of transfer to the test surface on locomotion, animals were allowed to recover from the noxious mechanical stress (transfer with a wire pick) for $10 \mathrm{~min}$ before being examined.
After $10 \mathrm{~min}$ at $20^{\circ} \mathrm{C}$, wild-type (N2) animals had a reversal rate of $4.25 \pm 0.73$ reversals/minute, when maintained at $20^{\circ} \mathrm{C}$ for another $10 \mathrm{~min}$ this rate slightly increased (5.25 \pm 0.69 reversals/ minute (Figures 2A, B, black bars)). Immediately after the temperature drop to $15^{\circ} \mathrm{C}$ wild-type animals reduced their rate of reversals (a 3.4 -fold reduction to $1.25 \pm 0.4$ reversals/minute; Figure 2A), a reduction likely to represent an escape response i.e., increased forward speed, reduced pauses and reduced reversals (Cohen et al., 2012). This reduction is maintained for $10 \mathrm{~min}$ at the noxious temperature of $15^{\circ} \mathrm{C}$ (Figure 2B). Since, the response observed using this assay differs from the response to lower temperature seen in the previous assay [Figure $\mathbf{1}$ and (Cohen et al., 2014)] we examined whether it represents a PVDmediated response to temperature downshift. For this, we examined animals lacking PVD neurons [-PVD animals (Albeg et al., 2011)]. Indeed, -PVD animals exhibit a weaker, statistically insignificant reduction in reversals immediately after the temperature downshift (Figure 2A) and no reduction in the rate of reversals following $10 \mathrm{~min}$ at $15^{\circ} \mathrm{C}$ (Figure 2B). Thus, reduced rate of reversals following temperature downshift requires PVD neurons allowing us to use this assay to examine the role of GTL-1 in the immediate response to thermal stimuli. Indeed, this assay demonstrates the similarity between $g t l-1(l f)$ and -PVD animals in their immediate and enduring responses to temperature downshift (Figures 2A, B). Together, our results (Figures 1 and 2) demonstrate an important role for GTL-1 in the response to cold temperatures.

\section{GTL-1 and the Response to Mechanical Stimuli}

GTL-1 was shown to amplify optogenetic stimuli to PVD, a result suggesting that it functions downstream to nocisensors (Husson et al., 2012). Thus, GTL-1 is unlikely to function
A

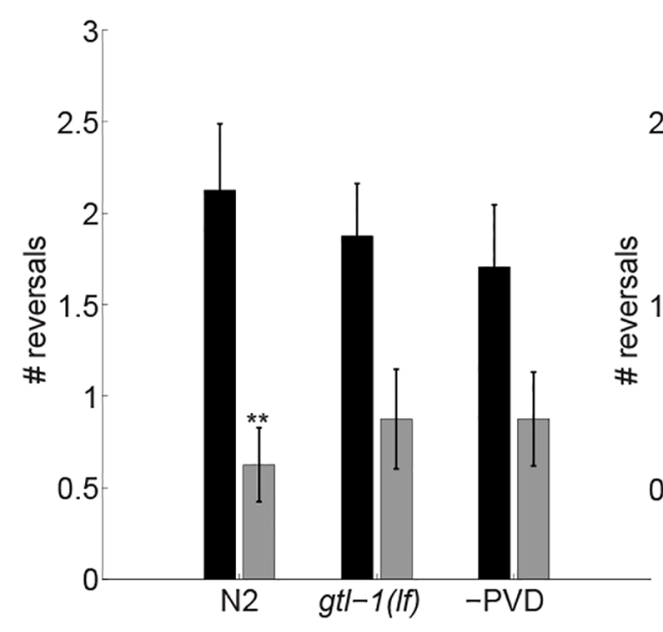

B

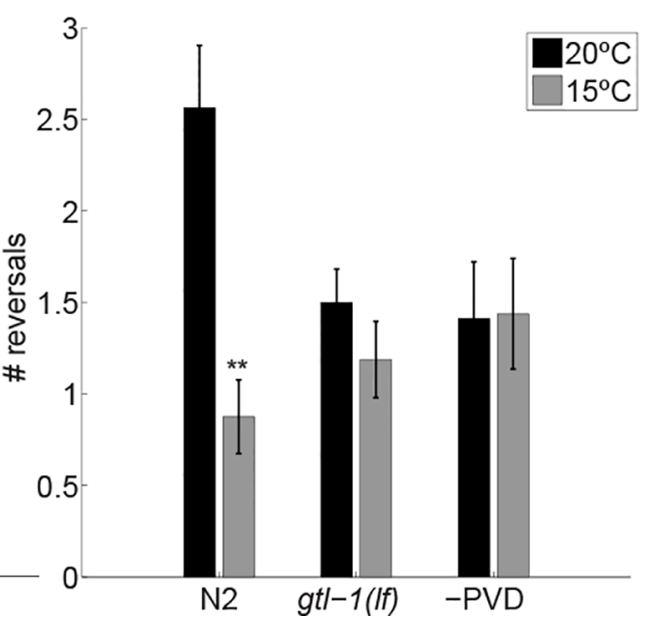

FIGURE 2 | GTL-1 is required for the immediate and enduring response to cold temperatures. Number of reversals was counted over 30 sec immediately after the temperature drop $(\mathbf{A})$ and 10 min later $\mathbf{( B )} . \mathrm{n}=16$ each. Significant differences relative to controls, maintained at $20^{\circ} \mathrm{C}(\mathrm{black}$ bars) throughout the experiments, were examined using two-way ANOVA **-p<0.01). 
specifically in the response to thermal stimuli and is likely to also affect the response to noxious mechanical stimuli. To examine the role of GTL-1 in the response to mechanical stimuli we first examined its effects on percent animals responding to single noxious mechanical stimulus (prodding with a platinum wire pick). Since this response depends on both PVD and touch receptor neurons (Way and Chalfie, 1989) we compared responses of mec-4(e1611) animals, in which touch receptor neurons degenerate (Driscoll and Chalfie, 1991), to responses of $g t l-1(l f) ; m e c-4(e 1611)$ lacking GTL-1 and touch receptor neurons. Using this assay we found a small but insignificant effect of GTL-1 on percent animals responding to noxious mechanical stimuli (Figure 3A). Thus $g t l-1$ is not essential for a reliable response to a single high-threshold mechanical stimulus.

Responses to noxious stimuli habituate to a lower extent relative to responses to similar non noxious stimuli; as shown when comparing responses to repeated optogenetic activation of PVD neurons relative to responses to repeated optogenetic activation of the low threshold touch receptor neurons (Husson et al., 2012). To examine whether GTL-1 is required for maintaining the response to repeated mechanical stimuli we compared decay in percent animals responding to repeated noxious mechanical stimuli between wild-type, gtl-1(lf) and PVD animals. Results of this analysis are consistent with PVD neurons being needed for maintaining the response to repeated mechanical stimuli as, animals lacking PVD neurons show a stronger decay in the response to repeated prodding with a platinum wire pick relative to wild type animals (Figure 3B). Our results, however, show that GTL-1 is not required for maintaining the response to repeated noxious mechanical stimuli as its responses to repeated prodding are indistinguishable from these of wild-type animals (Figure 3B).
Results in Figure 3 show that GTL-1 is not required for producing a reliable behavioral response to high threshold mechanical stimuli or for maintaining a reliable response to repeated high threshold mechanical stimuli. However, these assays did not examine magnitude of the response to such stimuli. To examine whether GTL-1 affects response magnitude to mechanical stimuli, we imaged locomotion of animals for $20 \mathrm{~min}$ following transfer of the animal to a new plate with a platinum wire pick; this assay provides a sensitive and quantitative measure for roles of genes and neurons in the response to mechanical stimuli (Cohen et al., 2012). Results of this analysis show that both N2 and gtl-1(lf) animals show similar reduction of forward speed over time and a similar ratio between speed at the start point and the end point (1.59 and 1.68 , respectively). However, the starting speed of gtl-1(lf) is lower then that of wild-type animals (Figure 4A). Analysis of two other locomotion parameters, percent time moving forward and percent time pausing (Figures $\mathbf{4 B}, \mathbf{C}$ ) show a similar starting point but larger changes over time relative to wild-type. To better understand the role of PVD expressed GTL-1 in locomotion following noxious mechanical stimuli we examined animals following PVD-specific knockdown of gtl-1 expression, as described above (Figures 1D-F). Results of this analysis show reduced initial speed following transfer of the animal with a platinum wire pick, a difference that is maintained 3 to $4 \mathrm{~min}$ following transfer (Figure 4D). Other parameters examined show no difference between wild-type animals and transgenic animals (Figures 4E, F). These results demonstrate a role for PVD-expressed GTL-1 in determining the magnitude of the escape response (enhanced forward speed) following a noxious mechanical stimulus. Other effects of GTL-1 on locomotion in this assay are likely to depend on its expression in yet unidentified cells. Previously published results show that
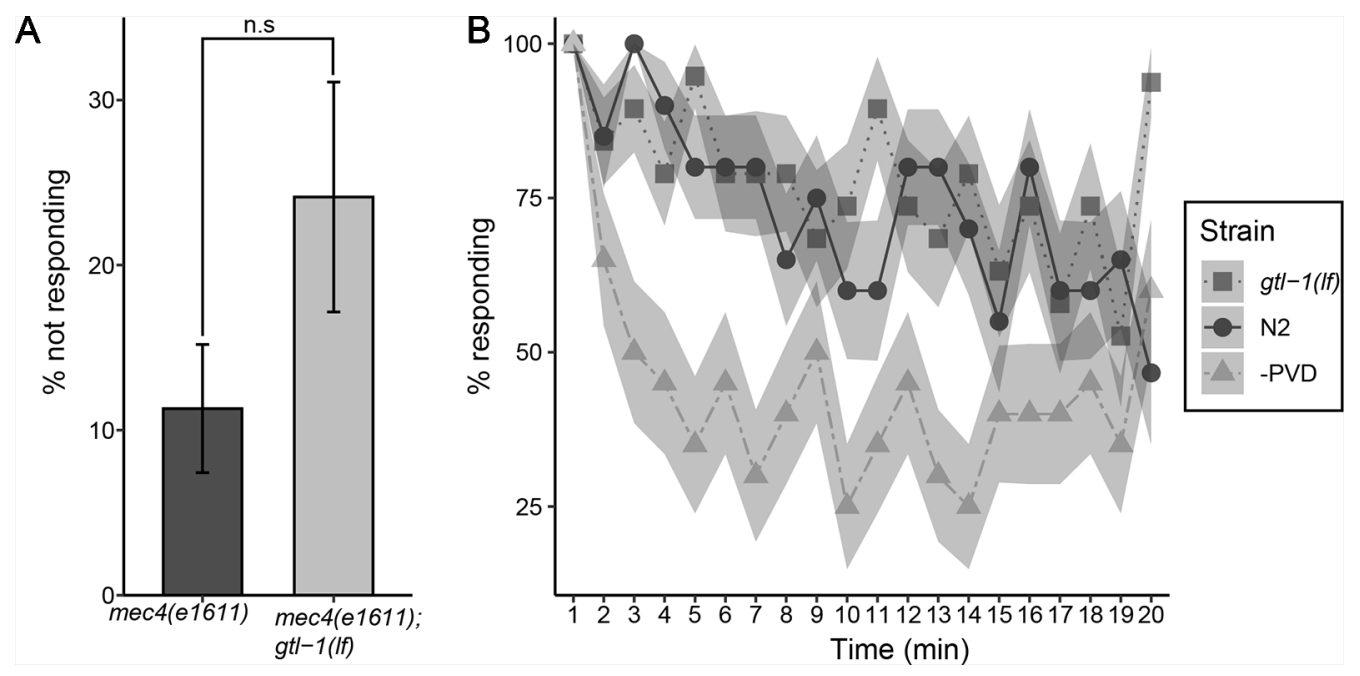

FIGURE 3 | GTL-1 is not essential for the response to single or repeated mechanical stimuli. (A) Percent animals not responding to a single high threshold mechanical stimuli in mec-4(e1611); gtl-1(If) and in mec-4(1611) animals (20 and 11 plates, respectively, 10 animals each plate). Statistical analysis was performed using n.s, $p=0.086$. (B) Responses to repeated high threshold mechanical stimuli in gtl-1(If) animals $(n=20)$, wild-type (N2, $n=20)$, and $-P V D(n=20)$ animals. 

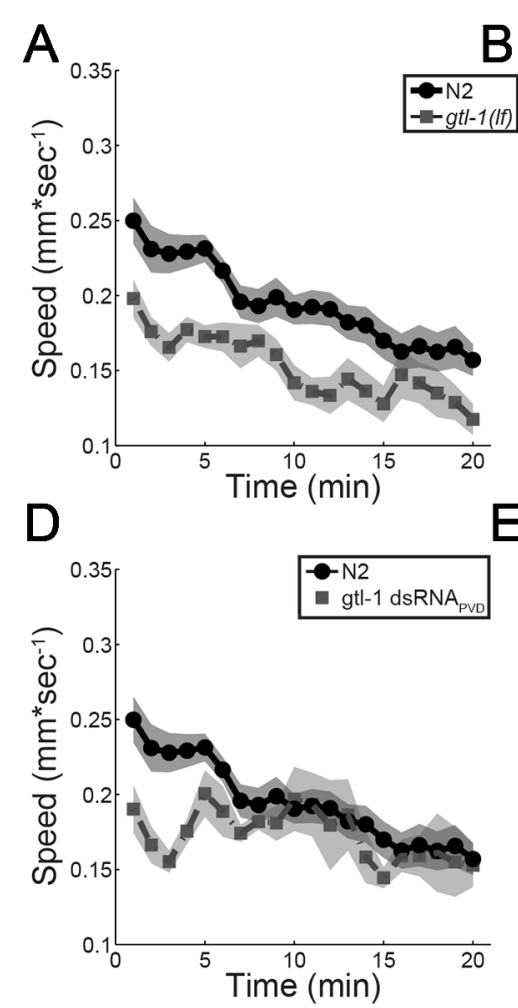
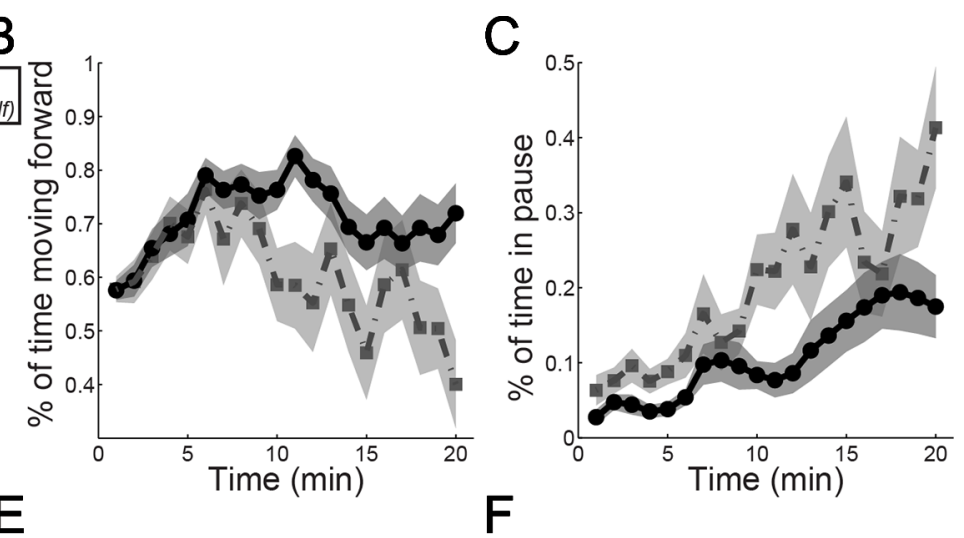

$F$
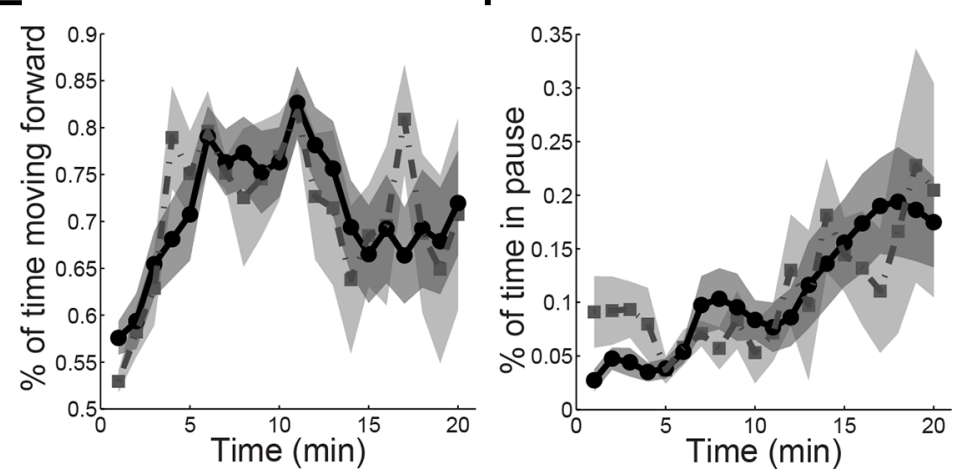

FIGURE 4 | GTL-1 affects the magnitude of the response to noxious mechanical stimuli. Immediate and enduring responses to a noxious mechanical stimulus of wild-type (N2, $N=19$, circles) compared to gtl-1(If) ( $\mathrm{n}=13$, squares) $(\mathbf{A}-\mathbf{C})$ and wild-type (N2, $n=19$, circles) compared to gtl-1 dsRNAPVD ( $\mathrm{n}=5$, squares) (D-F).

animals lacking PVD show a similar reduction in the escape response, relative to wild-type animals, i.e. reduced speed immediately following transfer with a wire pick, a reduction that lasts for a few min (Cohen et al., 2012). Thus, GTL-1 is likely to be an important determinant of this PVD-mediated escape response to noxious mechanical stimuli.

\section{Heterologous Expression of GTL-1}

Behavioral analysis (above) together with the previously published optogenetic analysis (Husson et al., 2012) show that GTL-1 functions in PVDs to enhance responses to multiple modalities. Previous studies suggest that GTL-1 might be a calcium-activated non-selective cation channel (CAN) (Xing et al., 2008; Xing and Strange, 2010), a mode of gating similar to that of TRPM4 and TRPM5 (Launay et al., 2002; Hofmann et al., 2003). Activation by cytosolic calcium may explain the role of GTL-1 in amplifying responses to multiple distinct stimuli activating PVDs.

To examine whether internal calcium activates GTL-1dependent currents we used the multi-channel inside-out configuration of the patch clamp technique in HEK293 cells; excised patches were exposed to $\mathrm{Ca}^{2+}$ at different membrane voltages; this analysis showed voltage and internal calcium dependent gating of GTL-1 (Figures 5A, B). Specifically, in cells expressing GTL-1 and in the presence of internal calcium we observed outwardly rectifying currents (Figure 5A). Although, these currents were small they were significantly larger then currents seen in the absence of calcium (Figure 5B). No difference was observed between effects of $0.1 \mathrm{mM}$ and 1 $\mathrm{mM}$ calcium on current amplitudes (Figure 5B); a result consistent with results obtained in the C. elegans intestine where GTL-1 and GON-2 function together to produce calcium oscillations and the defecation rhythm; these calcium oscillation were attributed to calcium having two opposing effects on these channels; activation at low concentrations and inhibition at higher concentrations (Estevez and Strange, 2005). Importantly, activation of GTL-1 by internal calcium enables its activation by receptors and channels affecting cytosolic calcium levels, directly or indirectly (Figure 6).

In the intestine GTL-1 was suggested to function downstream to PLC and $\mathrm{IP}_{3}$, known to function as part of signaling pathways leading to calcium release from internal stores (Xing and Strange, 2010). To examine whether $G_{\alpha} q$-dependent signaling, leading to PLC activation and $\mathrm{IP}_{3}$ signaling, activates GTL-1 we used a Designer Receptor Exclusively Activated by Designer Drugs (DREADD) developed for expression in HEK293 cells (Lee et al., 2014; Roth, 2016). Previously published results show that in HEK293 cells CNO-dependent currents are only detected when this DREADD receptor is co-expressed with a TRP channel and are abolished in the presence of a PLC inhibitor 
A

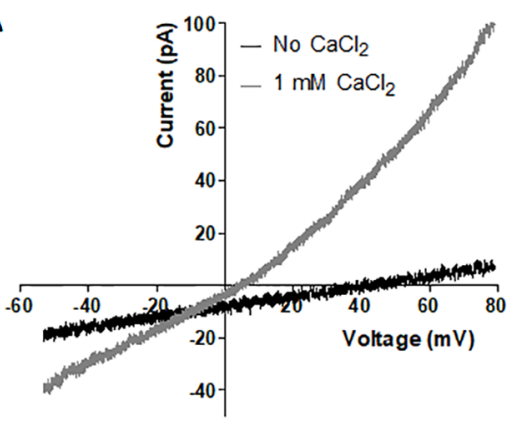

C

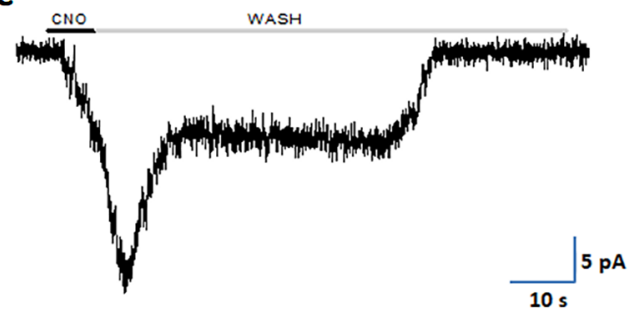

B

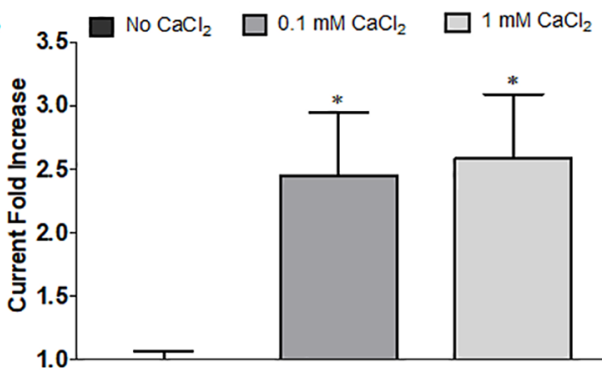

D DREADD-Gq + GTL1

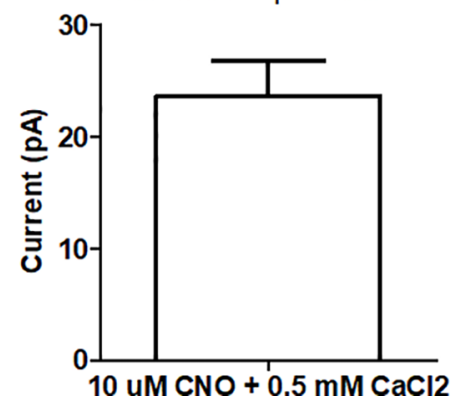

FIGURE 5 | Internal calcium and Goq activity gate GTL-1. GTL-1 was expressed in HEK293 cells. (A and B) Calcium enhances GTL-1 dependent currents. (A) Representative IV recording with and without calcium applied to the inner side of the excised inside-out patch. (B) Fold effect on current amplitudes of Calcium applied to the excised inside-out patch at $+80 \mathrm{mV}$. Effects of calcium are significant, $p<0.05$, $(n=8)$, one-way ANOVA with correction for multiple variables (C and D) GTL-1 is activated by DREADD-Goq. (C) Representative response. (D) Average response, $n=5$. Of note, no currents are detected in the absence of CNO or in cells not expressing GTL-1.
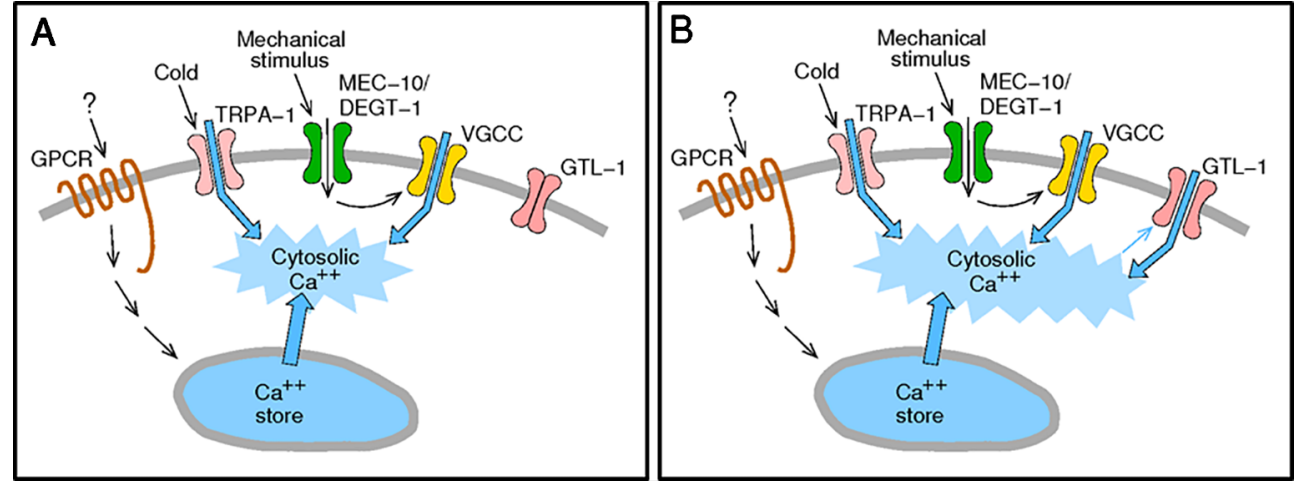

FIGURE 6 | Activation of GTL-1 by internal calcium enables amplification of sensory stimuli. (A) Sensory stimuli via nocisensor activation directly (TRPA-1) or indirectly (GPCRs and MEC-10/DEGT-1) elevate cytosolic calcium. (B) Elevated cytosolic calcium activates GTL-1 channels to amplify the calcium signal.

(Kumar et al., 2017). Thus, results (Figures 5C, D) showing reproducible CNO-activated currents when co-expressing GTL1 with this DREADD receptor are a clear indication for GTL-1 being activated downstream to $G_{\alpha} q$-dependent signaling and are consistent with results obtained in the C. elegans intestine (Xing and Strange, 2010).

\section{DISCUSSION}

GTL-1, a member of the TRPM family of ion channels, was previously shown to amplify behavioral responses to optogenetic activation of PVD (Husson et al., 2012). Here, we set out to characterize the role of GTL-1in PVD-mediated responses to 
noxious, physiological, stimuli. PVD cells mediate the response to temperature downshift, a response requiring PVD expression of TRPA-1 (Chatzigeorgiou et al., 2010). Our results demonstrate similar defects in the behavioral responses to cold temperatures in $g t l-1(l f)$ animals, trpa-1(lf) animals, and animals lacking PVD. Analysis of the PVD mediated response to mechanical stimuli, however, shows milder GTL-1-dependent behavioral effects. Specifically our results show reduced response magnitude to a noxious mechanical stimulus. But, no significant reduction in the reliability of the response (percent animal responding) to a single or to repeated mechanical stimuli. To understand the mechanism enabling effects of GTL-1 on responses to thermal, mechanical, and optogenetic stimuli, we examined its gating using electrophysiology in HEK293 cells. Results of this analysis show that internal calcium and Goq-signaling gate this channel. Thus, GTL-1 like other TRPM channels, and as described in Figure 6, enables amplification of responses to receptors and channels that, directly or indirectly, enhance internal calcium levels (Launay et al., 2002; Zurborg et al., 2007).

Our results suggest that GTL-1 is mainly required for responses to immediate and enduring cold temperatures while having a relatively minor role in the response to high threshold mechanical stimuli. Non-sensor channels that are required for a specific modality within a polymodal nociceptor have previously been reported. For example, inhibition of the voltage-gated potassium channel, $\mathrm{k}_{\mathrm{v}} 1.1$, leads to severe mechanical allodynia (i.e., lowers the threshold and causes non-painful stimuli to be sensed as painful) without affecting the threshold for noxious heat (Hao et al., 2013); the voltage-gated sodium channel, $\mathrm{Na}_{\mathrm{v}} 1.8$, on the other hand, has been shown to specifically affect transmission of cold sensation, by being the only voltage-gated sodium channel active at low temperatures (Matthews et al., 2006; Zimmermann et al., 2007). Additional analysis of GTL-1 channel's properties is needed to better understand its modality specific effects.

In mammals, the TRPM family consists of 8 different channels TRPM1-8. Members of this family are implicated in various biological processes, e.g., regulation of $\mathrm{Ca}^{2+}$ oscillations following T-lymphocyte activation (TRPM4) (Launay et al., 2004), regulation of magnesium absorption in kidneys and the intestine (TRPM6) (Voets et al., 2004; Vriens et al., 2011), gustatory transduction (TRPM5) and innocuous and noxious thermal sensing (TRPM3 and 8)(Zhang et al., 2003; Bautista et al., 2007; Dhaka et al., 2007; Vriens et al., 2011). C. elegans expresses three TRPM channels, gon-2, gtl-1, and gtl-2. These channels express widely including the intestine, the gonad, excretory cells, and the nervous system. Functions of these channels include, maintaining $\mathrm{Ca}^{2+}$ and $\mathrm{Mg}^{2+}$ homeostasis in the intestines and in extracellular fluids (Teramoto et al., 2005; Stawicki et al., 2011), controlling defecation rhythm (Kwan et al., 2008; Xing et al., 2008), and regulating the onset and continuation of post-embryonic mitotic cell divisions in the somatic gonad (Sun and Lambie, 1997; West et al., 2001). Our results combined with the results in (Husson et al., 2012) show for the first time a neuronal function for GTL-1; enhancing responses to noxious stimuli.
Previous work showed that in the C. elegans intestine GTL-1 functions together with GON-2 to produce a strong inwardly rectifying current, $\mathrm{I}_{\mathrm{ORCa}}$. The role of GTL-1 in this current was deduced from comparing currents in gtl-1, gon-2, or gon-2;gtl-1 loss of function animals to wild-type animals. These studies suggested that GTL-1 has a minor role in $\mathrm{I}_{\mathrm{ORCa}}$ and that it is likely to be activated by cytosolic $\mathrm{Ca}^{2+}$ and to function downstream of PLC signaling (Teramoto et al., 2005; Xing et al., 2008; Xing and Strange, 2010). This study, for the first time, characterizes properties of the GTL-1 channel in a heterologous expression system. Results of this analysis are consistent with the in-vivo results, showing mild outward rectification of the GTL-1 dependent currents, activation by internal calcium and by $\mathrm{G} \alpha \mathrm{q}$ signaling. Importantly, our findings suggest a mechanism, summarized in Figure 6, whereby GTL-1 is activated downstream of channels, receptors and signaling pathways whose activation directly or indirectly increases cytosolic calcium; thus enabling GTL-1 dependent enhancement of multiple and distinct sensory modalities as demonstrated by its effects on behavioral responses to thermal and mechanical stimuli.

\section{DATA AVAILABILITY STATEMENT}

The datasets generated for this study are available on request to the corresponding author.

\section{AUTHOR CONTRIBUTIONS}

EC performed behavioural analysis, cloned gtl-1 cDNA into mammalian cell expression vector, organized results for article, and participated in writing the article. RK performed and analyzed heterologous expression studies. TZ set up the system enabling imaging and analysis of the response to rapid temperature downshift, performed the experiments, and analyzed them. AP supervised the heterologous expression studies and participated in writing the article. MT supervised behavioral analysis and wrote the article.

\section{FUNDING}

This work was supported by the Prusiner-Abramsky research award to MT, the Israel Science Foundation (Grants 1444/16 to AP), the Brettler Center and David R. Bloom Center, School of Pharmacy (The Hebrew University of Jerusalem; to AP), a Jerusalem Brain Committee Postdoctoral Fellowship (to RK).

\section{ACKNOWLEDGMENTS}

We thank the C. elegans Genetics Stock Center for strains, Howard Baylis for the gtl-1 cDNA, and Anatoly Shapochnikov for assistance with setting up the immediate cooling device. 


\section{REFERENCES}

Albeg, A., Smith, C. J., Chatzigeorgiou, M., Feitelson, D. G., Hall, D. H., Schafer, W. R., et al. (2011). C. elegans multi-dendritic sensory neurons: morphology and function. Mol. Cel Neurosci. 46, 308-317. doi: 10.1016/j.mcn. 2010.10.001

Babcock, D. T., Landry, C., and Galko, M. J. (2009). Cytokine signaling mediates UV-induced nociceptive sensitization in Drosophila larvae. Curr. Biol. 19, 799806. doi: 10.1016/j.cub.2009.03.062

Bautista, D. M., Siemens, J., Glazer, J. M., Tsuruda, P. R., Basbaum, A. I., Stucky, C. L., et al. (2007). The menthol receptor TRPM8 is the principal detector of environmental cold. Nature 448, 204-208. doi: 10.1038/nature05910

Caterina, M. J., and Julius, D. (1999). Sense and specificity: a molecular identity for nociceptors. Curr. Opin. In Neurobiol. 9, 525-530. doi: 10.1016/S0959-4388 (99)00009-4

Caterina, M. J., Leffler, A., Malmberg, A. B., Martin, W. J., Trafton, J., PetersesZeitz, K. R., et al. (2000). Impaired nociceptrion and pain sensation in mice lacking the capsaicin receptor. Science 288, 306-313. doi: 10.1126/ science.288.5464.306

Chatzigeorgiou, M., Yoo, S., Watson, J. D., Lee, W. H., Spencer, W. C., Kindt, K. S., et al. (2010). Specific roles for DEG/ENaC and TRP channels in touch and thermosensation in C. elegans nociceptors. Nat. Neurosci. 13, 861-868. doi: 10.1038/nn.2581

Clapham, D. (2003). TRP channels as cellular sensors. Nature 426, 519-524. doi: 10.1038/nature02196

Cohen, E., Yemini, E., Feitelson, D. G., Schaffer, W. R., and Treinin, M. (2012). Locomotion analysis identifies roles of mechanosensory neurons in governing locomotion dynamics of C. elegans. J. Exp. Biol. 215, 3639-3648. doi: 10.1242/ jeb.075416

Cohen, E., Chatzigeorgiou, M., Husson, S. J., Steuer-Costa, W., Gottschalk, A., Schafer, W. R., et al. (2014). C. elegans nicotinic acetylcholine receptors are required for nociception. Mol. Cell Neurosci. 59, 85-96. doi: 10.1016/ j.mcn.2014.02.001

Dhaka, A., Viswanath, V., and Patapoutian, A. (2006). Trp ion channels and temperature sensation. Annu. Rev. Neurosci. 29, 135-161. doi: 10.1146/ annurev.neuro.29.051605.112958

Dhaka, A., Murray, A. N., Mathur, J., Earley, T. J., Petrus, M. J., and Patapoutian, A. (2007). TRPM8 is required for cold sensation in mice. Neuron 54, 371-378. doi: 10.1016/j.neuron.2007.02.024

Driscoll, M., and Chalfie, M. (1991). The mec-4 gene is a member of a family of Caenorhabditis elegans genes that can mutate to induce neuronal degeneration. Nature 349, 588-593. doi: 10.1038/349588a0

Esposito, G., Schiavi, E. D., Bergamasco, C., and Bazzicalupo, P. (2007). Efficient and cell specific knock-down of gene function in targeted C. elegans neurons. Gene 395, 170-176. doi: 10.1016/j.gene.2007.03.002

Estevez, A. Y., and Strange, K. (2005). Calcium feedback mechanisms regulate oscillatory activity of a TRP-like Ca2+ conductance in C. elegans intestinal cells. J. Physiol. 567, 239-251. doi: 10.1113/jphysiol.2005.091900

Geron, M., Kumar, R., Zhou, W., Faraldo-Gómez, J. D., Vásquez, V., and Priel, A. (2018). TRPV1 pore turret dictates distinct DkTx and capsaicin gating. Proc. Natl. Acad. Sci. U.S.A. 115, E11837-E11846. doi: 10.1073/pnas.1809662115

Hao, J., Padilla, F., Dandonneau, M., Lavebratt, C., Lesage, F., Noël, J., et al. (2013). Kv1.1 channels act as mechanical brake in the senses of touch and pain. Neuron 77, 899-914. doi: 10.1016/j.neuron.2012.12.035

Hofmann, T., Chubanov, V., Gudermann, T., and Montell, C. (2003). TRPM5 is a voltage-modulated and $\mathrm{Ca}(2+)$-activated monovalent selective cation channel. Curr. Biol. 13, 1153-1158. doi: 10.1016/s0960-9822(03)00431-7

Husson, S. J., Steuer Costa, W., Wabnig, S., Stirman, J. N., Watson, J. D., Spencer, W. C., et al. (2012). Optogenetic analysis of a nociceptor neuron and network reveals modulatory ion channels acting downstream of nociceptive sensors. Curr. Biol. 22, 743-752. doi: 10.1016/j.cub.2012.02.066

Julius, D. (2013). TRP channels and pain. Annu. Rev. Cell Dev. Biol. 29, 355-384. doi: 10.1146/annurev-cellbio-101011-155833

Kamath, R. S., Fraser, A. G., Dong, Y., Poulin, G., Durbin, R., Gotta, M., et al. (2003). Systematic functional analysis of the Caenorhabditis elegans genome using RNAi. Nature 421, 231-237. doi: 10.1038/nature01278
Kumar, R., Hazan, A., Geron, M., Steinberg, R., Livni, L., Matzner, H., et al. (2017). Activation of transient receptor potential vanilloid 1 by lipoxygenase metabolites depends on PKC phosphorylation. FASEB J. 31, 1238-1247. doi: 10.1096/fj.201601132R

Kwan, C. S., Vázquez-Manrique, R. P., Ly, S., Goyal, K., and Baylis, H. A. (2008). TRPM channels are required for rhythmicity in the ultradian defecation rhythm of C. elegans. BMC Physiol. 8, 11. doi: 10.1186/1472-6793-8-11

Launay, P., Fleig, A., Perraud, A. L., Scharenberg, A. M., Penner, R., and Kinet, J. P. (2002). TRPM4 is a Ca2+-activated nonselective cation channel mediating cell membrane depolarization. Cell 109, 397-407. doi: 10.1016/s0092-8674(02) 00719-5

Launay, P., Cheng, H., Srivatsan, S., Penner, R., Fleig, A., and Kinet, J. P. (2004). TRPM4 regulates calcium oscillations after T cell activation. Science 306, 13741377. doi: 10.1126/science. 1098845

Lee, H. M., Giguere, P. M., and Roth, B. L. (2014). DREADDs: novel tools for drug discovery and development. Drug Discovery Today 19, 469-473. doi: 10.1016/ j.drudis.2013.10.018

Matthews, E. A., Wood, J. N., and Dickenson, A. H. (2006). Na(v) 1.8-null mice show stimulus-dependent deficits in spinal neuronal activity. Mol. Pain 2, 5. doi: 10.1186/1744-8069-2-5

Priel, A., and Silberberg, S. D. (2004). Mechanism of ivermectin facilitation of human P2X4 receptor channels. J. Gen. Physiol. 123, 281-293. doi: 10.1085/ jgp. 200308986

Roth, B. L. (2016). DREADDs for Neuroscientists. Neuron 89, 683-694. doi: 10.1016/j.neuron.2016.01.040

Smith, E. S., and Lewin, G. R. (2009). Nociceptors: a phylogenetic view. J. Comp. Physiol. A 195, 1089-1106. doi: 10.1007/s00359-009-0482-z

Smith, C. J., Watson, J. D., Spencer, W. C., OÕBrien, T., Cha, B., Albeg, A., et al. (2010). Time-lapse imaging and cell-specific expression profiling reveal dynamic branching and molecular determinants of a multi-dendritic nociceptor in C. elegans. Dev. Biol. 345, 18-33. doi: 10.1016/j.ydbio.2010.05.502

Stawicki, T. M., Zhou, K., Yochem, J., Chen, L., and Jin, Y. (2011). TRPM channels modulate epileptic-like convulsions via systemic ion homeostasis. Curr. Biol. 21, 883-888. doi: 10.1016/j.cub.2011.03.070

Sun, A. Y., and Lambie, E. J. (1997). gon-2, a gene required for gonadogenesis in Caenorhabditis elegans. Genetics 147, 1077-1089.

Teramoto, T., Lambie, E. J., and Iwasaki, K. (2005). Differential regulation of TRPM channels governs electrolyte homeostasis in the C. elegans intestine. Cell Metab. 1, 343-354. doi: 10.1016/j.cmet.2005.04.007

Tsalik, E. L., Niacaris, T., Wenick, A. S., Pau, K., Avery, L., and Hobert, O. (2003). LIM homeobox gene-dependent expression of biogenic amine receptors in restricted regions of the C. elegans nervous system. Dev. Biol. 263, 81-102. doi: 10.1016/s0012-1606(03)00447-0

Voets, T., Nilius, B., Hoefs, S., van der Kemp, A. W., Droogmans, G., Bindels, R. J., et al. (2004). TRPM6 forms the Mg2+ influx channel involved in intestinal and renal Mg2+ absorption. J. Biol. Chem. 279, 19-25. doi: 10.1074/jbc. M311201200

Vriens, J., Owsianik, G., Hofmann, T., Philipp, S. E., Stab, J., Chen, X., et al. (2011). TRPM3 is a nociceptor channel involved in the detection of noxious heat. Neuron 70, 482-494. doi: 10.1016/j.neuron.2011.02.051

Way, J. C., and Chalfie, M. (1989). The mec-3 gene of Caenorhabditis elegans requires its own product for maintained expression and is expressed in three neuronal cell types. Genes Dev. 3, 1823-1833. doi: 10.1101/gad.3. 12 a. 1823

West, R. J., Sun, A. Y., Church, D. L., and Lambie, E. J. (2001). The C. elegans gon2 gene encodes a putative TRP cation channel protein required for mitotic cell cycle progression. Gene 266, 103-110. doi: 10.1016/s0378-1119(01)00373-0

Woolf, C. J., and Ma, Q. (2007). Nociceptors-noxious stimulus detectors. Neuron 55, 353-364. doi: 10.1016/j.neuron.2007.07.016

Xing, J., and Strange, K. (2010). Phosphatidylinositol 4,5-bisphosphate and loss of PLCgamma activity inhibit TRPM channels required for oscillatory $\mathrm{Ca} 2+$ signaling. Am. J. Physiol. Cell Physiol. 298, C274-C282. doi: 10.1152/ ajpcell.00394.2009

Xing, J., Yan, X., Estevez, A., and Strange, K. (2008). Highly Ca2+-selective TRPM channels regulate IP3-dependent oscillatory Ca2+ signaling in the C. elegans intestine. J. Gen. Physiol. 131, 245-255. doi: 10.1085/jgp.200709914 
Zhang, Y., Hoon, M. A., Chandrashekar, J., Mueller, K. L., Cook, B., Wu, D., et al. (2003). Coding of sweet, bitter, and umami tastes: different receptor cells sharing similar signaling pathways. Cell 112, 293-301. doi: 10.1016/s0092-8674 (03)00071-0

Zimmermann, K., Leffler, A., Babes, A., Cendan, C. M., Carr, R. W., Kobayashi, J., et al. (2007). Sensory neuron sodium channel Nav1.8 is essential for pain at low temperatures. Nature 447, 855-858. doi: 10.1038/nature05880

Zurborg, S., Yurgionas, B., Jira, J. A., Caspani, O., and Heppenstall, P. A. (2007). Direct activation of the ion channel TRPA1 by Ca2+. Nat. Neurosci. 10, 277-279. doi: $10.1038 / \mathrm{nn} 1843$
Conflict of Interest: The authors declare that the research was conducted in the absence of any commercial or financial relationships that could be construed as a potential conflict of interest.

Copyright (C) 2020 Cohen, Kumar, Zinger, Priel and Treinin. This is an open-access article distributed under the terms of the Creative Commons Attribution License (CC BY). The use, distribution or reproduction in other forums is permitted, provided the original author(s) and the copyright owner(s) are credited and that the original publication in this journal is cited, in accordance with accepted academic practice. No use, distribution or reproduction is permitted which does not comply with these terms. 